\title{
Abstract Potential Operators on Hilbert Space
}

(Dedicated to Professor Yasuo Akizuki on his 70th birthday)

By

\section{Kôsaku YosıdA*}

Let $X$ be a (real or complex) Hilbert space. A linear operator $V$ with its domain $D(V)$ and range $R(V)$ both strongly dense in $X$ is called an abstract potential operator (see K. Yosida [2], p. 412) if the inverse $V^{-1}$ exists in such a way that

$$
A=-V^{-1}
$$

is the infinitesimal generator of a one-parameter semi-group of class $\left(C_{0}\right)$ of linear contraction operators on $X$ into $X$. The purpose of the present note is to prove the following existence theorem. (Hereafter, we shall denote by $S^{a}$ the strong closure of a subset $S$ of $X$.)

Theorem. Let $U$ be a linear operator satisfying three conditions:

$$
\begin{aligned}
& D(U)^{a}=X, \\
& R(U)^{a}=X,
\end{aligned}
$$

(4) $U$ is accretive, that is, $\operatorname{Re}(U f, f) \geqq 0 \quad$ for every $f \in D(U)$.

Then there exists at least one abstract potential operator $V$ which is a closed linear accretive extension of $U ; V$ might coincide with $U$.

Proof. The proof is given in two steps. The first is to construct a maximal accretive extension $V$ of $U$ by virtue of R. S. Phillips' theory of

Received March 2, 1972.

* Dept. Math., Gakushuin Univ., 1-5-1, Mejiro, Toshima-ku, Tokyo 171, Japan. 
Cayley transform (cf. B. Sz.-Nagy and C. Foias [1], p. 167). The second is to prove that this $V$ is an abstract potential operator by making use of the abelian ergodic theorem for pseudo-resolvents (see K. Yosida [2], p. 215).

The First Step. For every $\lambda>0$ and $f \in D(V)$, we have, by (4),

$$
\begin{gathered}
\|\lambda U f+f\|^{2}=(\lambda U f+f, \lambda U f+f)=\|\lambda U f\|^{2}+2 \operatorname{Re}(\lambda U f, f)+\|f\|^{2} \\
\geqq\|\lambda U f\|^{2}+\|f\|^{2} \geqq\|\lambda U f\|^{2}-2 \operatorname{Re}(\lambda U f, f)+\|f\|^{2}=\|\lambda U f-f\|^{2} .
\end{gathered}
$$

Hence the inverse $(\lambda U+I)^{-1}$ exists and moreover, the Cayley transform $C$ defined through

$$
C \cdot(U f+f)=(U f-f)
$$

is a contraction operator mapping $R(U+I)$ onto $R(U-I)$. Let us define a bounded linear extension $\hat{C}$ of $C$ :

(7) through continuity on $R(U+I)^{a}$, and through putting $\hat{C} \cdot g=0$ on the orthogonal complement of $R(U+I)$.

This everywhere defined contraction operator $\hat{C}$ cannot admit eigenvalue one. Assume the contrary and let $\hat{C} \cdot f_{0}=f_{0}$ with $\left\|f_{0}\right\|=1$. Then its adjoint operator $\hat{C}^{*}$, which is also a contraction, must satisfy $\hat{C}^{*} \cdot f_{0}=f_{0}$ because

$$
\begin{aligned}
& \left\|\hat{C}^{*} \cdot f_{0}-f_{0}\right\|^{2}=\left\|\hat{C}^{*} \cdot f_{0}\right\|^{2}-2 \operatorname{Re}\left(\hat{C}^{*} \cdot f_{0}, f_{0}\right)+\left\|f_{0}\right\|^{2} \\
& \leqq\left\|f_{0}\right\|^{2}-2 \operatorname{Re}\left(f_{0}, \hat{C} \cdot f_{0}\right)+\left\|f_{0}\right\|^{2}=1-2+1=0 .
\end{aligned}
$$

Thus we obtain, by (6) and (7),

$$
\left(f_{0},(U-I) f\right)=\left(f_{0}, \hat{C} \cdot(U+I) f\right)=\left(\hat{C}^{*} \cdot f_{0},(U+I) f\right)=\left(f_{0},(U+I) f\right),
$$

hence $\left(f_{0}, f\right)=0$ and so $f_{0}=0$ by (2).

Therefore the inverse $(I-\hat{C})^{-1}$ exists and so we can define a linear operator $V$ through

$$
V \cdot(I-\hat{C}) f=(I+\hat{C}) f
$$


$V$ is an extension of $U$. In fact, we have, by $(6),(I-C)=I-(U-I)$ $(U+I)^{-1}=2(U+I)^{-1}$, that is, $U=(I+C)(I-C)^{-1}$, proving that $V$ is an extension of $U$. Here the existence of $(I-C)^{-1}$ is assured by that of $(I-\hat{C})^{-1}$. We can prove that $V$ is accretive. For, by putting $f$ $=(I-\hat{C})^{-1} g$ and observing (8) and the contraction property of $\hat{C}$, we obatin

$$
R e(V g, g)=\operatorname{Re}((I+\hat{C}) f,(I-\hat{C}) f)=\|f\|^{2}-\|\hat{C} \cdot f\|^{2} \geqq 0
$$

We can also prove, by (8) and the boundedness of the operator $\hat{C}$, that $V$ is a closed linear operator. Moreover, by (8), we have $(I+V)$ $=I+(I+\hat{C})(I-\hat{C})^{-1}=2(I-\hat{C})^{-1}$, and so we obtain the existence theorem

(9) $\quad R(V+I)=D(I-\hat{C})=X \quad$ (and also $R(\lambda V+I)=X$ whenever $\lambda>0)$.

Hence the accretive extension $V$ is maximal as regards its range $R(\lambda V+I)$ for $\lambda>0$.

The Second Step. We will show that $V$ is an abstract potential operator following after the proof of Theorem 2 on p. 414-415 in K. Yosida [2].

$V$ being accretive, we have, as in (5), $\left\|\lambda V f+f^{\prime}\right\| \geqq\|\lambda V f\|$ for every $f \in D(V)$ and $\lambda>0$. Hence, by (9), we can define a bounded linear operator

$$
J_{\lambda}=V(\lambda V+I)^{-1}
$$

satisfying

$$
\left\|\lambda J_{\lambda}\right\| \leqq 1
$$

It is easy to see that $J_{\lambda}$ is a pseudo-resolvent, i.e.,

$$
J_{\lambda}-J_{\mu}=(\mu-\lambda) J_{\lambda} J_{\mu} .
$$

Therefore, by (11), we can apply the abelian ergodic theorem to the effect that

$$
R\left(J_{\mu}\right)^{a}=\left\{x \in X ; s-\lim _{\lambda \uparrow \infty} \lambda J_{\lambda} x=x\right\} \quad \text { for all } \mu>0
$$




$$
R\left(I-\mu J_{\mu}\right)^{a}=\left\{x \in X ; s-\lim _{\lambda \downarrow 0} \lambda J_{\lambda} x=0\right\} \quad \text { for all } \mu>0 .
$$

By $R(V)^{a}=R(U)^{a}=X$, we have $R\left(J_{\mu}\right)^{a}=X$ by (10) and so, by (11) and (12), the null space of $J_{\lambda}$ consists of zero vector only, independently of $\lambda>0$. Hence $J_{\lambda}$ is the resolvent of a linear operator, i. e.,

$$
J_{\lambda}=(\lambda I-A)^{-1}, \quad \text { where } A=\lambda I-J_{\lambda}^{-1} \text { is independent of } \lambda>0 \text {. }
$$

We have thus $D(A)^{a}=R\left(J_{\mu}\right)^{a}=X$ and so, by (11), the operator $A$ is the infinitesimal generator of a contraction semi-group of class $\left(C_{0}\right)$. We can also prove that $R(A)^{a}=X$. For, we have, by (10) and (15),

$$
(\lambda I-A) J_{\lambda}(\lambda V f+f)=\lambda V f+f=(\lambda I-A) V f=\lambda V f-A V f,
$$

that is,

$$
-A V f=f \quad \text { whenever } f \in D(V) \text {, }
$$

proving that $R(A)^{a}=D(V)^{a}=D(U)^{a}=X$. Thus, by (14) and $A J_{\mu}=$ $=\left(\mu J_{\mu}-I\right)$, we obtain $s-\lim _{\lambda \downarrow 0} \lambda J_{\lambda} f=0$ for all $f \in X$. This implies that the inverse $A^{-1}$ exists. In fact, the condition $A f_{0}=0$ is equivalent to $\lambda(\lambda I-A)^{-1} f_{0}=f_{0}$ and hence $f_{0}=\underset{\lambda \downarrow 0}{s-\lim _{\lambda}} \lambda J_{\lambda} f_{0}=0$.

Thus $-A^{-1}$ is an abstract potential operator. On the other hand, (16) shows that the inverse $V^{-1}$ exists. Hence, by $(\lambda I-A)=J_{\lambda}^{-1}=$ $=(\lambda V+I) V^{-1}=\lambda I+V^{-1}$, we obtain $-A=V^{-1}$, completing the proof of our Theorem.

Remark. We shall verify (2), (3) and (4) for Newtonian and logarithmic potentials

$$
\begin{aligned}
& (U f)(y)=\int_{R^{n}} K_{n}(|y-z|) f(z) d z \quad(n \geqq 2), \\
& K_{n}(r)=r^{2-n} \quad \text { for } n \geqq 3, \quad \text { and } \quad K_{2}(r)=\log r^{-1} .
\end{aligned}
$$

The proof of $D(U)^{a}=R(U)^{a}=X=L^{2}\left(R^{n}\right)$ can be obtained by making use of the fact that, for $0<\delta_{1}<\delta_{2}$, 


$$
u_{x, \delta_{1}, \delta_{2}}(y)=\left(K_{n}\left(\delta_{1}\right)-K_{2}\left(\delta_{2}\right)\right)^{-1} \int_{R^{n}} K_{n}(|y-z|)\left(d \nu_{x, \delta_{1}}(z)-d \nu_{x, \delta_{2}}(z)\right)
$$

is continuous in $y$ satisfying

$$
\begin{array}{cl}
u_{x, \delta_{1}, \delta_{2}}(y)=1 & \text { if }|y-x| \leqq \delta_{1}, \\
=0 & \text { if }|y-x| \geqq \delta_{2}, \\
0<u_{x, \delta_{1}, \delta_{2}}(y)<1 & \text { if } \delta_{1}<|y-x|<\delta_{2} .
\end{array}
$$

Here $\nu_{x, \delta}$ is the unit measure uniformly distributed over the hypersurface of the sphere of centre $x$ and radius $\delta$ in $R^{n}$.

The Gauss-Frostmann energy inequality

$$
\int_{R^{n}}(U f)(y) \cdot \overline{f(y)} d y \geqq 0 \quad(n \geqq 2)
$$

holds good whenever $f \in L^{2}\left(R^{n}\right)$ is of compact support satisfying $\int_{R^{n}} f(y) d y=0$. It is easy to prove that such $f^{\prime}$ 's constitute a strongly dense subset of $L^{2}\left(R^{n}\right)$.

Another Treatment of the Second Step (Added on 20 April, 1972). As in the above proof of the non-existence of the eigenvalue 1 for the operator $\hat{C}$, we can show that $\hat{C} \cdot f_{0}=-f_{0}$ implies $\hat{C}^{*} \cdot f_{0}=-f_{0}$ and hence $\left(f_{0}, U f\right)=0$, proving by (3) the non-existence of the eigenvalue -1 for $\hat{C}$. Thus $V=(I+\hat{C})(I-\hat{C})^{-1}$ given by (8) admits the inverse $V^{-1}=$ $=(I-\hat{C})(I+\hat{C})^{-1}$. Hence we can prove that $V$ is an abstract potential operator without appealing to the abelian ergodic theorem.

Remark (added during the proof). On reading the pre-print, Prof. K. Sato gave interesting comments and extensions. See his paper to appear.

\section{References}

[1] Sz.-Nagy, B. and C. Foias, Harmonic Analysis of Operators on Hilbert Space, North Holland Publ. Co., 1970.

[2] Yosida, K., Functional Analysis, the Third Ed., Springer-Verlag, 1971. 
